\title{
Physics of fluid transport in hybrid biporous capillary wicking microstructures
}

Saitej Ravi, Ramanathan Dharmarajan and Saeed Moghaddam*

*Author to whom correspondence should be addressed. Email: saeedmog@ufl.edu

\begin{tabular}{|l|r|}
\hline Title & Page Number \\
\hline SEM image of a segmented in-plane hybrid wick & S1 \\
\hline Image of chamber utilized in dryout threshold measurements & S1 \\
\hline Heat loss in a typical wick structure & S3 \\
\hline Schematic depicting the behavior of an evaporating meniscus in an out-of-plane & S4 \\
\hline hybrid wick & S5 \\
\hline Estimation of mesh permeability & \\
\hline Temperature variation of out-of-plane hybrid wicks & \\
\hline
\end{tabular}




\section{Supporting Information for Publication}

1. Segmented in-plane hybrid wick

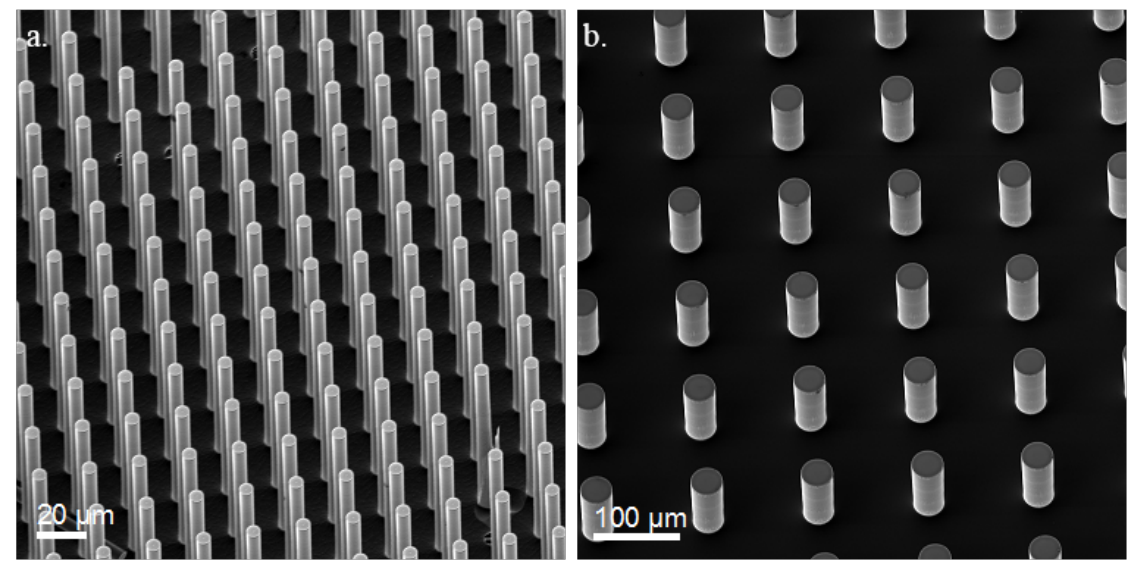

Figure S1. Scanning electron micrograph (SEM) of (a) evaporator and (b) adiabatic sections of B-2 wick

2. Test chamber

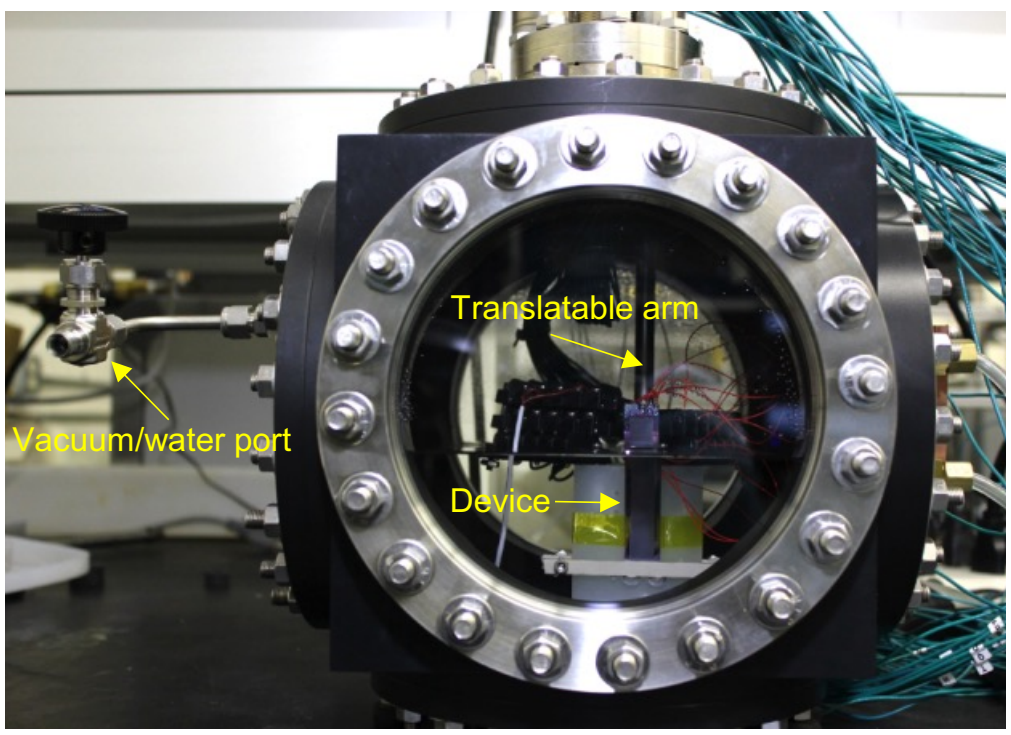

Figure S2. An image of the stainless steel chamber with a test device mounted on the polycarbonate block 
3. Heat loss in a typical wick structure

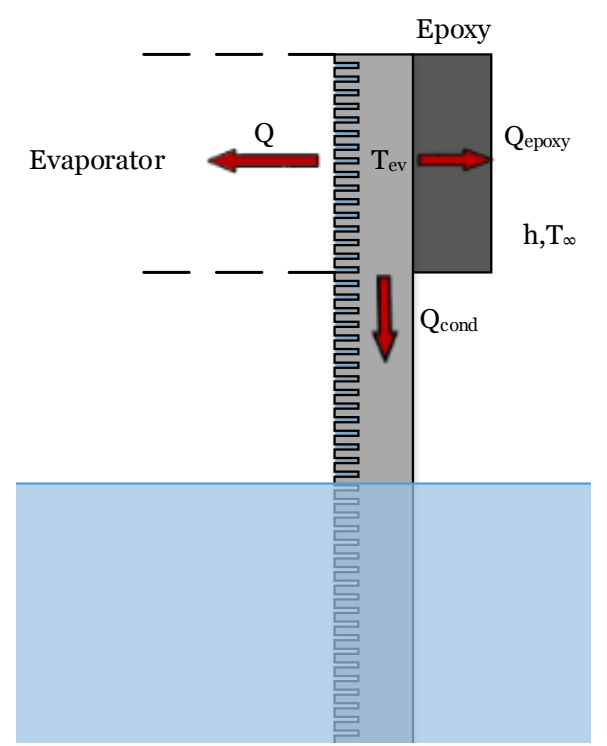

Figure S3. Depiction of heat loss due to conduction and convection heat transfer mechanisms. 
4. Evaporating meniscus in an out-of-plane hybrid wick

a.

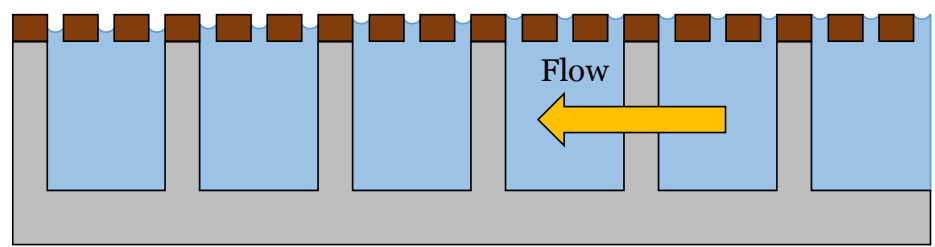

b.

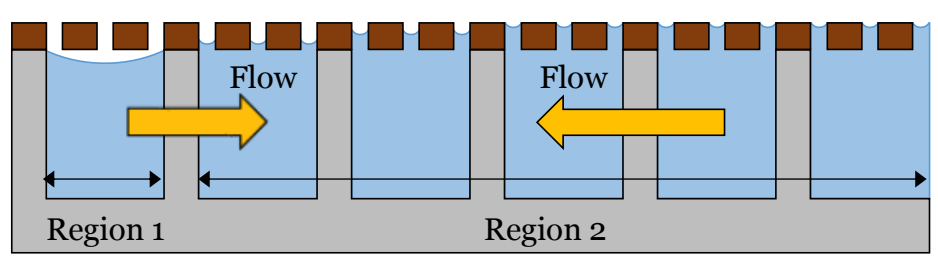

c.
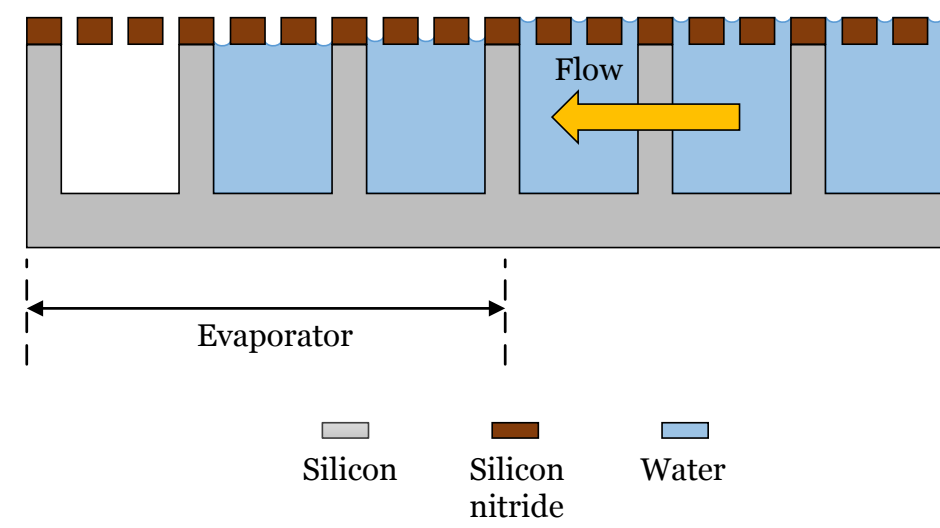

Figure S4. Schematic representation of the behavior of evaporating liquid menisci and dryout in an outof-plane hybrid wick. a. configuration of an evaporating liquid meniscus constrained in the mesh; b. liquid meniscus configuration just prior to dryout divides the evaporator into two distinct regions based on the position of the meniscus relative to the mesh; c. Spontaneous local dryout in the evaporator. 
5. Estimation of mesh permeability

An order-of-magnitude estimate of the permeability of the stainless steel mesh used in type E hybrid wicks was determined using the rate of rise method. The tests were performed in the test chamber to prevent liquid evaporation. The mesh was suspended over the pool of water on the translatable arm and the arm was lowered until the base of the mesh was just below the liquid surface. The rise of water along the mesh was recorded at $30 \mathrm{fps}$.

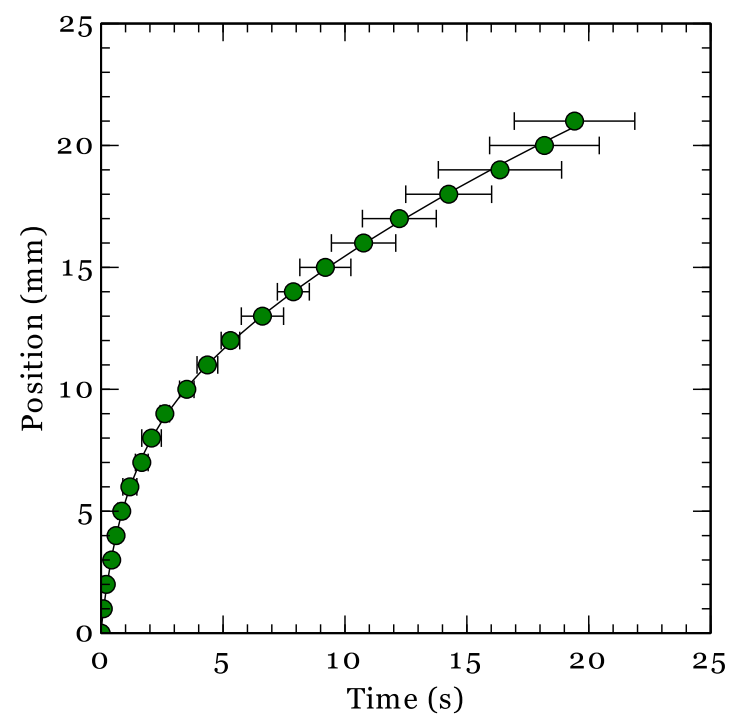

Figure S5. Interface position vs. time data obtained from the rate of rise experiments on the stainless steel mesh used in the type E hybrid wicks.

The interface position vs. time data extracted from the rate of rise experiments is plotted in Figure S5. The data was fit to a liquid rise model proposed by Rogacs et al., ${ }^{\mathrm{S} 1}$ which includes the effect of

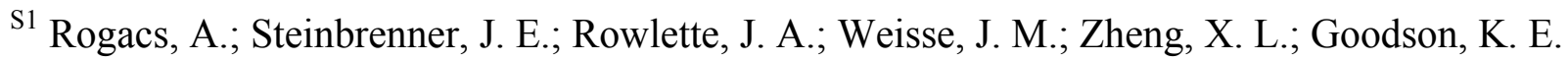
Characterization of the Wettability of Thin Nanostructured Films in the Presence of Evaporation. J. Colloid Interface Sci. 2010, 349, 354-360. 
gravity on the liquid rise and neglects the effects of liquid evaporation (Eq. S-1). Using the properties of water at $24{ }^{\circ} \mathrm{C}$, the permeability of the mesh was determined to be $1.50 \mathrm{E}-11 \mathrm{~m}^{2}$. This value is an order of magnitude smaller than the permeability of the homogeneous wick, which was measured to be $2.01 \mathrm{E}-10$ $\mathrm{m}^{2}$. Therefore, it can be assumed that the liquid transport through the type E hybrid wicks occurs primarily through the micropillar arrays and not through the mesh.

$l(t)=\frac{P_{c} K}{\mu \phi}\left[1+W\left(-\exp \left(-1-\frac{\rho^{2} g^{2} K}{\phi P_{c}} t\right)\right)\right]$

Here, $W($ ) represents the Lambert $\mathrm{W}$ function.

6. Temperature variation in out-of-plane hybrid wicks

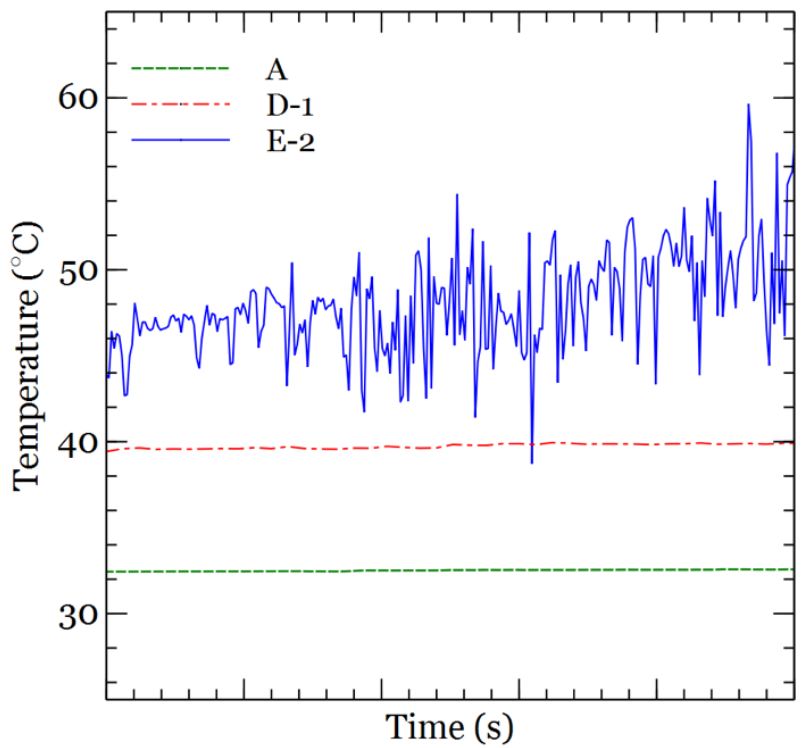

Figure S6. Temporal variations of temperature just prior to dryout on wicks A, D-1 and E-2 at a wicking length of $3 \mathrm{~cm}$.

Local evaporator temperatures were recorded using the thin film sensors embedded within the heater of each device. The temperature data recorded just prior to dryout on wicks A, D-1 and E-2 are 
presented in Figure S6. The steady state values for wicks A and D-1 indicate the presence of thin film evaporation at the liquid vapor interface. The oscillations in temperature of the E-2 wick occur due to nucleate boiling on the evaporator wall. 